\title{
DISTRIBUTION OF STOCHASTIC FORCES IN GRAVITATIONALLY CLUSTERED SYSTEM OF GALAXIES
}

\author{
ELIANI ARDI ${ }^{1,2}$ AND SHOGO INAGAKI ${ }^{1}$ \\ ${ }^{1}$ Department of Astronomy, Kyoto University \\ Kyoto 606-01, Japan \\ ${ }^{2}$ Department of Astronomy, Institute of Technology \\ Bandung, Indonesia
}

\section{Motivation:}

We are interested in examining the influence of nearest neighbor galactic encounters in gravitationally clustered systems by using cosmological $\mathrm{N}$ body simulation.

\section{Research:}

We investigate the evolution of the stochastic force distribution which comes from all the galaxies in the system and the force from the nearest galaxy before and during gravitational clustering of galaxies in the expanding universe, through numerical experiments. N-body simulations were done by using a COMOVEV code.

\section{Conclusions:}

The force acting on each galaxy in gravitationally clustered systems in the expanding universe is almost entirely due to the gravitational attraction of the nearest neighbor. Nearest neighbor galactic encounters play the main role in the dynamics of galaxy clustering in the expanding universe.

\section{Implications:}

In a gravitationally clustered system, each encounter can be treated as a two-body encounter representing the perturber galaxy and its nearest neighbor galaxy. The use of a softening parameter in the collisionless $\mathrm{N}$ body simulation method may not be well suited to study the dynamics of galaxy clustering because the forces from the nearest neighbors may be neglected. 